\title{
Vertical Resolution Requirements in Atmospheric Simulation
}

\author{
William C. Skamarock, Chris SNyder, AND JosePh B. KlemP \\ National Center for Atmospheric Research, Boulder, Colorado \\ SANG-HUN PARK \\ Yonsei University, Seoul, South Korea
}

(Manuscript received 19 February 2019, in final form 24 April 2019)

\begin{abstract}
The role of vertical mesh spacing in the convergence of full-physics global atmospheric model solutions is examined for synoptic, mesoscale, and convective-scale horizontal resolutions. Using the MPAS-Atmosphere model, convergence is evaluated for three solution metrics: the horizontal kinetic energy spectrum, the Richardson number probability density function, and resolved flow features. All three metrics exhibit convergence in the free atmosphere for a $15-\mathrm{km}$ horizontal mesh when the vertical grid spacing is less than or equal to $200 \mathrm{~m}$. Nonconvergence is accompanied by noise, spurious structures, reduced levels of mesoscale kinetic energy, and reduced Richardson number peak frequencies. Coarser horizontal mesh solutions converge in a similar manner but contain much less noise than the $15-\mathrm{km}$ solutions for coarse vertical resolution. For convective-scale resolution simulations with $3-\mathrm{km}$ cell spacing on a variable-resolution mesh, solution convergence is almost attained with a vertical mesh spacing of $200 \mathrm{~m}$. The boundary layer scheme is the dominant source of vertical filtering in the free atmosphere. Although the increased vertical mixing at coarser vertical mesh spacing depresses the kinetic energy spectra and Richardson number convergence, it does not produce sufficient dissipation to effectively halt scale collapse. These results confirm and extend the results from a number of previous studies, and further emphasize the sensitivity of the energetics to the vertical mixing formulations in the model.
\end{abstract}

\section{Introduction}

Over the last 50 years, horizontal mesh spacing in global numerical weather prediction (NWP) and climate model configurations has decreased by much more than an order of magnitude. For example, Lindzen and FoxRabinovitz (1989, their Fig. 1) show that global model configurations in the $1970 \mathrm{~s}$ used $1.5^{\circ}$ to $+5^{\circ}$ mesh spacing compared with the $1 / 10^{\circ}$ spacing used in many operational global NWP configurations today. Vertical mesh spacing in these configurations, however, has increased by much less than an order of magnitude, from a few kilometers in the 1970s and 1980s [see Lindzen and FoxRabinovitz (1989), their Figs. 1 and 2] to a typical value of $0.5 \mathrm{~km}$ in the free troposphere today. Convectionpermitting NWP model configurations use horizontal mesh spacing almost an order of magnitude smaller than their global counterparts, yet the configurations have very similar vertical grid spacing. While there have been

Corresponding author: William C. Skamarock, skamaroc@ucar.edu some studies addressing the question of appropriate vertical mesh spacing for a given horizontal mesh spacing, there is neither a consensus answer nor a common approach to framing the question, particularly for NWP and climate atmospheric model applications.

In this paper we use global NWP model simulation results to examine solution convergence as a function of vertical resolution. This study is motivated in part by the understanding that the cost of horizontal resolution scales approximately as the inverse of the horizontal mesh spacing to the third power [i.e., cost $\sim \Delta x^{-3}$ ], given the need to decrease the time step proportional to the decrease in the horizontal mesh spacing. The cost of vertical resolution, however, scales as $\Delta z^{-1}$, or at worst $\Delta z^{-2}$ if the time step must be decreased to maintain stability. If solution accuracy in existing model configurations is limited by the vertical resolution, then it may be optimal to increase the vertical resolution before increasing the horizontal resolution given the cost differential. Perhaps more importantly, insufficient vertical resolution in model configurations can have deleterious 
effects, for example where solutions exhibit spurious but seemingly resolved flow structures and/or poorly resolved structures at the grid scale (noise).

One of the earliest studies that examined the question of appropriate vertical mesh spacing for a given horizontal mesh spacing was that of Lindzen and FoxRabinovitz (1989). Using quasigeostrophic theory, they postulated that

$$
\Delta z \approx(f / N) \Delta x
$$

which in general terms can be interpreted as requiring the mesh-spacing ratio $\Delta z / \Delta x$ to be approximately the ratio of the scale height of the atmosphere to the Rossby radius. Lindzen and Fox-Rabinovitz (1989) noted that global models of the day were vertically underresolved in the midlatitudes based on this analysis, although they did not show results concerning the consequences of this underresolution.

Around the same time Pecnick and Keyser (1989) presented results from idealized simulations of $2 \mathrm{D}$ frontogenesis that led them to relate the required resolution ratio to the frontal slope:

$$
\Delta z \leq \Delta z_{\text {opt }}=m_{\text {slope }} \Delta x,
$$

where the frontal slope $m_{\text {slope }}$ in their simulations was approximately $5 \times 10^{-3}$. The use of insufficient vertical resolution based on (2) results in the appearance of spurious gravity waves in the vicinity of the fronts. Similar results regarding spurious waves have been addressed in subsequent in studies, including those by Persson and Warner (1991), Snyder et al. (1993), Lean and Clark (2003), Iga et al. (2007). Given that the ratio of the atmospheric scale height and the Rossby radius is itself an estimate of the frontal slope, the estimates of the mesh spacing ratio $\Delta z / \Delta x$ given by (1) and (2) are similar, ranging between approximately $1 / 100$ and $1 / 200$.

The dynamical arguments used to justify resolution ratios in the midlatitudes do not extend to the tropics. Lindzen and Fox-Rabinovitz (1989) examined gravity wave dynamics to address resolution requirements here, and they suggest that the most severe constraints on vertical resolution will appear at critical layers where a gravity wave's vertical wavelength will collapse. Lindzen and Fox-Rabinovitz (1989) invoke dissipation to prevent this collapse and suggest that the necessary resolution could be estimated by

$$
\Delta z \approx\left(\sigma_{i} / N\right) \Delta x,
$$

where the damping rate $\sigma_{i}=\tau^{-1}$ the damping time scale. Damping halts the vertical scale collapse occurring as waves approach a critical layer. Using second-order vertical mixing leads to the relation between the vertical eddy viscosity and the damping time scale, $\sigma_{i}=$ $\left(\nu / \Delta z^{2}\right)$. They estimate that a value of $\nu=100 \mathrm{~m}^{2} \mathrm{~s}^{-1}$ would be consistent with coarse-mesh models having $(\Delta x \sim 100 \mathrm{~km})$, a damping time scale of a few hours and a vertical mesh of a kilometer or less, but they also recognize that this eddy viscosity is much larger than diagnosed and used in most parameterizations of vertical mixing in the free atmosphere. The relevance of this gravity wave critical-layer result to vertical resolution requirements is not obvious, but it does raise the question of the role of atmospheric model dissipative processes in preventing vertical scale collapse. We will return to this issue in the discussion of our results.

Waite (2016) and Cullen (2017) suggest that, in addition to the QG-based requirement in (1), the buoyancy scale limits the vertical mesh spacing:

$$
\Delta z \ll L_{b}=2 \pi U / N .
$$

The term $L_{b}$ describes the thickness of vertical shear layers arising in stratified turbulence and typical atmospheric values for $L_{b}$ range from a few hundred meters to a kilometer (Waite 2016; Waite and Bartello 2004; Billant and Chomaz 2001). Waite (2016) used idealized turbulence simulations where vertical mesh spacings less than $200 \mathrm{~m}$ were needed to resolve the shear layers. The relevance of these idealized simulations to Earth's atmosphere is, however, somewhat uncertain given the very shallow slope of the mesoscale kinetic energy spectrum produced in the simulations. In a different study, Cullen (2017), using forecasts from the global Met Office model, produce similar estimates of the required vertical mesh spacing while acknowledging that the $40-\mathrm{km}$ horizontal mesh spacing used in his simulations does not resolve the mesoscale. Waite (2016) also suggested that the vertical resolution constraint may transition from (1) to (4) when the Rossby number exceeds $O(1)$, as found in rotating-stratified turbulence simulations (Lindborg 2005; Waite and Bartello 2006).

When inertia-gravity waves account for a significant portion of the flow, they can impose additional requirements on the vertical resolution. The required $\Delta z$ for inertia-gravity waves has received little attention in the literature, though Watanabe et al. (2015) visually examine inertia-gravity waves in the stratosphere in their simulations and find that 200-300-m vertical mesh spacing is needed before the waves appear resolved. A more general result follows from the $f$-plane dispersion relation for these waves, which implies that the ratio 
of the vertical wavenumber $m$ to the horizontal wavenumber $k$ satisfies

$$
m^{2} / k^{2}=\left(N^{2} / f^{2}\right)\left(\tilde{\omega}^{2} / f^{2}-1\right)^{-1},
$$

where $\tilde{\omega}$ is the wave's intrinsic frequency. For a given $k$, the required resolution then depends on the $\tilde{\omega}$ that characterizes the wave field. Waves with $\tilde{\omega}=\sqrt{2} f$ have $m / k=N / f$ and thus broadly the same resolution requirements as QG flows, namely $\Delta z / \Delta x \approx f / N$. As $\tilde{\omega}$ decreases toward the local inertial frequency $f$, however, $m$ becomes large, and the vertical scale and necessary $\Delta z$ approach zero.

Whether these resolution requirements are relevant then depends on the importance, or not, of inertiagravity waves in the flow. Inertia-gravity waves have been frequently observed in the stratosphere and upper troposphere, using a variety of measurements (e.g., Sato et al. 1997; Vaughan and Worthington 2007; Guest et al. 2000; Wang and Alexander 2010). In particular, intrinsic-frequency spectra for horizontal velocities in the stratosphere, which can be computed from the quasiLagrangian measurements provided by constant-pressure balloons, reveal a near-inertial peak (Hertzog et al. 2002). Model simulations also reveal inertia-gravity waves in the upper troposphere and lower stratosphere (e.g., Sato et al. 1999; Plougonven and Snyder 2007) and point to a role for inertia-gravity waves in determining the mesoscale kinetic energy spectrum (Waite and Snyder 2009; Burgess et al. 2013)

While most studies examining vertical resolution have only qualitatively examined simulation results, some studies have taken a more systematic approach. For example, Waite (2016) examines the convergence of the kinetic energy (KE) spectrum to quantitatively address vertical resolution requirements and to examine certain questions regarding model filter configurations. Cullen (2017) examines the convergence of model-estimated Froude numbers as a function of vertical resolution in the context of the role of stratified turbulence in controlling the resolution requirements. A few other studies, such as Brune and Becker (2013) and Waite and Snyder (2013), have looked at KE spectra while changing the vertical resolution but did not demonstrate spectra convergence. From a numerical analysis viewpoint, vertical resolution requirements can be cast as requirements for convergence of selected solution norms. The KE spectrum is an important norm because it measures the atmospheric flow energetics as a function of horizontal length scale, and its behavior (i.e., the slope of the spectrum as a function of horizontal wavenumber or wavelength) is tied to specific distinct dynamical regimes in atmospheric flow.
Of equal importance, the general character of the KE spectra has been observationally documented (Nastrom and Gage 1985; Lindborg 1999), and reproduced in atmospheric model simulations (e.g., Skamarock 2004; Hamilton et al. 2008).

In this paper we use the Model for Prediction Across Scales (MPAS; Skamarock et al. 2012) and its full-physics atmospheric global forecasts to examine the effect of vertical resolution on the convergence of the simulated KE spectrum and to examine the simulated atmospheric-flow structures and their convergence through the different vertical mesh spacings. We are not seeking a converged solution to the continuous fluid-flow equations in this study, rather we are seeking a converged discrete solution to a discrete atmospheric model where everything is held fixed except for the vertical mesh spacing. Importantly in these MPAS configurations, we hold fixed the horizontal mesh, the horizontal filters, and all the physics that do not depend on the vertical mesh spacing. In section 2 we describe the model and experimental design. We employ horizontal resolution consistent with current-day global operational NWP mesh spacing (15-km cell spacing); this configuration resolves some of the mesoscale regime in the atmosphere. In section 3 we examine the KE spectra produced in the simulations and we look at the flow structures in well-resolved and poorly resolved configurations. We see a consistent picture of the convergence of the KE spectrum and the flow structures. In section 4 we consider the role of model filters in limiting scale collapse in the simulations. Consistent with other studies, we find that vertical mixing (identified by Waite 2016) plays an important role in solution convergence. We begin to explore vertical resolution requirements when horizontal mesh spacing is extended to convectionpermitting resolutions in section 5 , and conclude with a summary and discussion in section 6 .

\section{Experimental configuration}

The MPAS-Atmosphere, version 5.1, is used in this study. ${ }^{1}$ The modeling system is described in Skamarock et al. (2012), and it employs unstructured spherical centroidal Voronoi horizontal meshes for the horizontal tiling of the sphere within its finite-volume formulation. Simulation results are presented for simulations using 120-, 60-, 30-, and 15-km cell-spacing uniform meshes, and a variable-resolution mesh with cell spacing that

\footnotetext{
${ }^{1}$ MPAS-Atmosphere is an open source model available at http:// mpas-dev.github.io.
} 


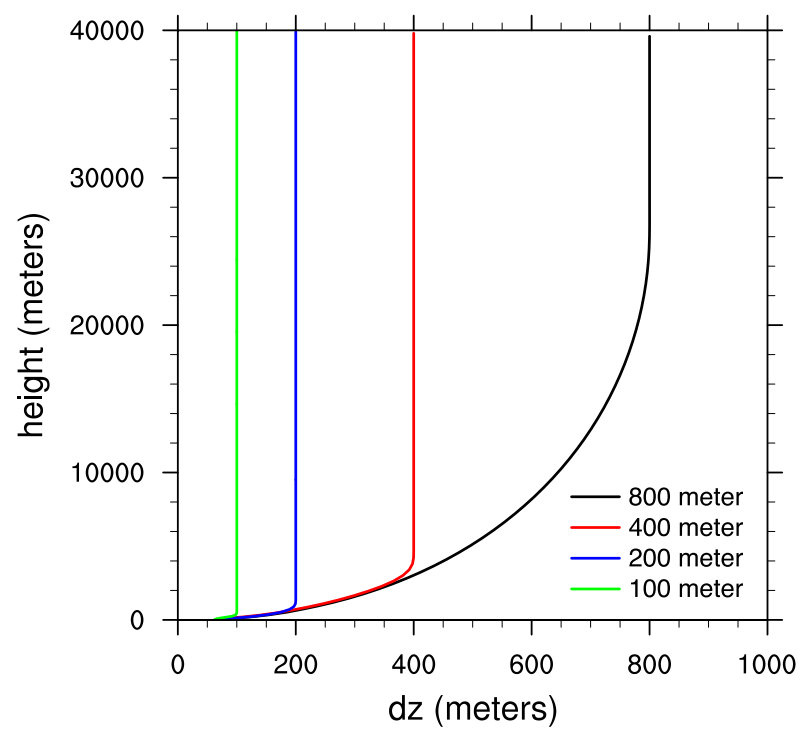

FIG. 1. Vertical grid spacing for the MPAS experiments. The 800-, 400-, 200-, and 100-m designations refer to the vertical grid spacing in the experiments

varies from 15 to $3 \mathrm{~km}$ in a high-resolution region. The MPAS-A configurations represent NWP configurations with complete physics suites; we use the mesoscalereference physics suite within the MPAS Version 5.1 release for simulations on the uniform meshes, and we replace the Tiedtke convective parameterization in that suite with the Grell-Freitas scale-aware convection scheme for the 15-3-km mesh simulations. All simulations are initialized at 0000 UTC 20 December 2016 from the GFS Final analysis, and 7-day simulations are produced in all the experiments.

The MPAS-A model top is set at $40 \mathrm{~km}$ for all the simulations, and a gravity wave absorbing layer Klemp et al. (2008) is used in the top $10 \mathrm{~km}$ of the simulated atmosphere. Four vertical level distributions are examined in these experiments, and the level distributions are shown in Fig. 1. The coarsest distribution represents a typical distribution in MPAS and other mesoscale models, for example in WRF (Skamarock and Klemp 2008). This distribution has a maximum vertical level spacing of $800 \mathrm{~m}$ over the upper half of the atmosphere. The other distributions limit the maximum level spacing to 400,200 , and $100 \mathrm{~m}$, and the mesh spacing in the transition between the vertically stretched mesh and the constant-level-spacing region is smoothed. In the remainder of this paper we denote each simulation by its horizontal and coarsest vertical mesh spacing, for example, the simulation using $100-\mathrm{m}$ vertical mesh from Fig. 1 and the $15-3-\mathrm{km}$ variable-resolution horizontal mesh is referred to as the $(15-3 \mathrm{~km}, 100 \mathrm{~m})$ simulation.

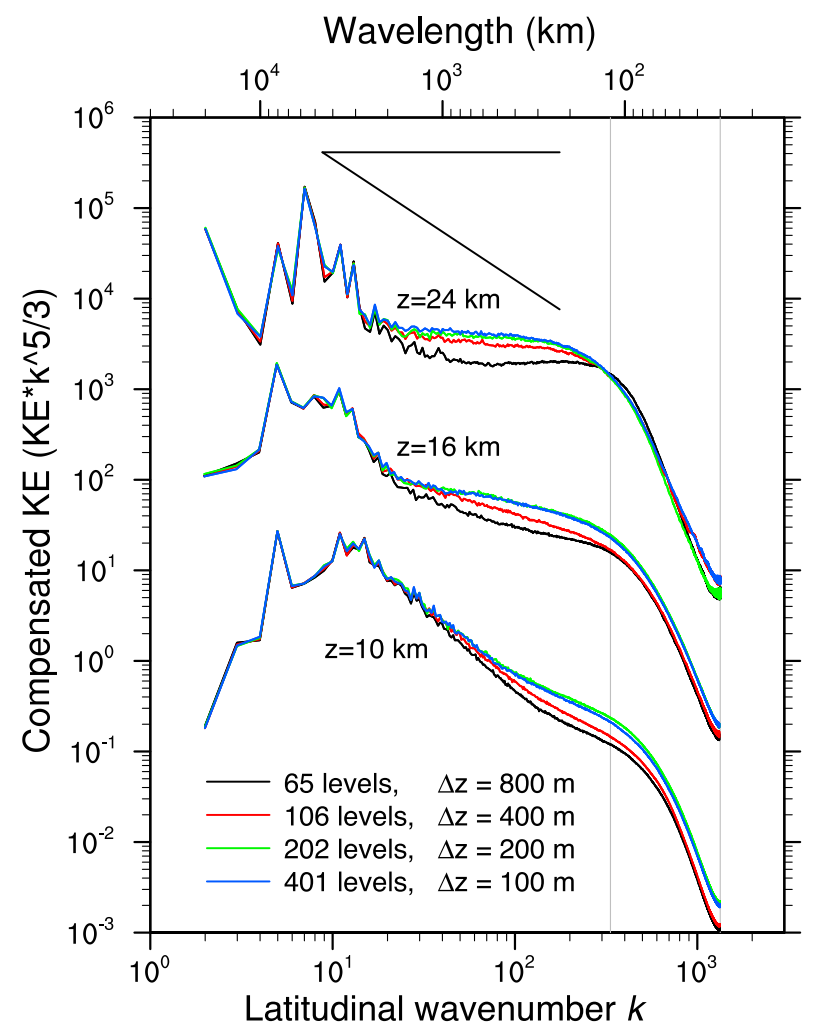

FIG. 2. Compensated horizontal KE $\left(\mathrm{KE} \times k^{5 / 3}\right)$ spectra from the $15-\mathrm{km}$ mesh simulations at $z=10,16$, and $24 \mathrm{~km}$ above sea level. For clarity, the $z=10-\mathrm{km}$ and $z=24-\mathrm{km}$ spectra are shifted two decades down and up, respectively. The $k^{-3}$ and $k^{-5 / 3}$ slopes are given at the top of the plot, and the $2 \Delta x$ are $8 \Delta x$ are plotted as gray vertical lines.

\section{Results from the $15-\mathrm{km}$ simulations}

\section{a. Kinetic energy spectra}

Figure 2 shows the compensated kinetic energy spectra (KE multiplied by the horizontal wavenumber $\left.k^{5 / 3}\right)$ as a function of $k$ and wavelength at $z=10$ (midlatitude upper troposphere), 16 (tropics upper troposphere) and $24 \mathrm{~km}$ (lower-middle stratosphere). These spectra are computed hourly, time averaged over simulation days 6 and 7, and displayed for the 15-km horizontal mesh simulations covering the 4 vertical grid distributions. The mesoscale transitions found in spectra computed from observations (Nastrom and Gage 1985; Lindborg 1999), from a more-steeply sloped $k^{-3}$ powerlaw behavior to a $k^{-5 / 3}$ behavior, begin at smaller wavenumbers at higher heights, with only a partial transition evident at $z=10$ and $z=16 \mathrm{~km}$. This behavior is consistent with the full-physics atmospheric simulation results in Skamarock et al. (2014, see their Fig. 9), where a less-than full transition is evident in the tropospheric spectrum for the $15-\mathrm{km}$ horizontal mesh results. 


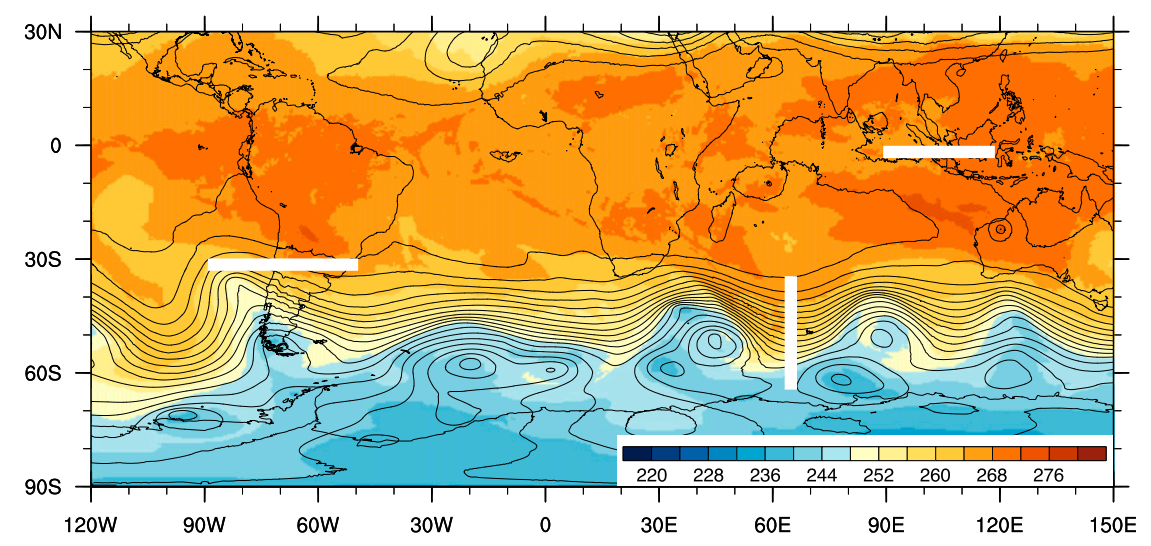

FIG. 3. 500-hPa heights and temperature from the $15-\mathrm{km} \Delta z=800-\mathrm{m}$ simulation at day 5 . The three cross sections are indicated by the thick white lines in South America, the Maritime Continent, and the south Indian Ocean.

The spectra results are also consistent with results from studies using other models, for example, the study of Hamilton et al. (2008, see their Fig. 8), where the transitions to a shallower sloped mesoscale spectrum occur at horizontal wavelengths of less than $1000 \mathrm{~km}$ in the troposphere and at progressively longer horizontal wavelengths with increasing height. Other aspects of the spectra are also comparable to results from earlier studies. The transitions in slope occur when the divergent component of the spectrum becomes similar in magnitude to the rotational component (not shown), as in (Skamarock 2004, see his Fig. 2) and Hamilton et al. (2008, see their Fig. 11). In the MPAS spectra, there is more downturn in the tails of the spectra compared with that from some model results in the literature (e.g., Brune and Becker 2013; Hamilton et al. 2008). This behavior is a model-configuration choice where poorly resolved waves are filtered so as to prevent any unphysical buildup of energy at the grid scale and contamination of the well-resolved portion of the spectrum. Interestingly, the spectra in Fig. 2 also reveals that the effects of model filters (downturn in the tails of the spectrum) are more pronounced at longer wavelengths at higher heights. This behavior may represent the cumulative effect of filtering upward-propagating waves, or it may indicate that the filtering is more aggressive than needed in the stratosphere.

The spectrum in Fig. 2 appears to be converged with $\Delta z \leq 200 \mathrm{~m}$ as indicated by the fact that the $(15 \mathrm{~km}$, $100 \mathrm{~m})$ and $(15 \mathrm{~km}, 200 \mathrm{~m})$ solutions are nearly identical at the three heights, while the $(15 \mathrm{~km}, 400 \mathrm{~m})$ and $(15 \mathrm{~km}, 800 \mathrm{~m})$ solutions show increasingly lower kinetic energy levels in the mesoscale region. The convergence is monotonic (i.e., the mesoscale KE increases as vertical resolution is increased and convergence is approached). This behavior is consistent with the results reported in
Waite (2016) in his stratified turbulence simulations using a vertical hyperviscosity-based mixing, but not with his results using other vertical mixing schemes. Brune and Becker (2013) find decreasing KE with increased vertical resolution in their experiments simulating the global atmosphere. This differing behavior of the KE spectrum may be caused by the role of model filters, which we discuss further in section 4 .

\section{b. Forecast features}

Figure 3 depicts the $500-\mathrm{hPa}$ height and temperature fields from the $(15 \mathrm{~km}, 800 \mathrm{~m})$ simulation at simulation day 5 . The white lines in the plot indicate the location of vertical cross sections; across the Andes in South America during a strong mountain-wave event, through a ridge in a Southern Hemisphere baroclinic wave, and through the Maritime Continent and Indian Ocean in the tropics in a region with significant convection and a strong easterly jet at upper levels.

Vertical cross sections of the horizontal divergence and potential temperature for the $(15 \mathrm{~km}, 100 \mathrm{~m})$ and $(15 \mathrm{~km}, 800 \mathrm{~m})$ simulations are plotted for the Andes cross section in Fig. 4 for day 6 in the simulations. Strong flow across the mountains is associated with a baroclinic wave, and the divergence fields show the deep waves over the mountains, strong wave breaking in the vicinity of the tropopause, and significant waves in the lower stratosphere. The sloping isentropes in the western part of domain are associated with the baroclinic wave. Vertically propagating inertia-gravity waves, indicated by shallowly sloping striations in the horizontal divergence that cross isentropes, are apparent above the baroclinic wave and also emanating from the breaking region of the mountain waves. The $(15 \mathrm{~km}, 800 \mathrm{~m})$ solution captures some of the features, but many of the waves are distorted, particularly the stratospheric waves 


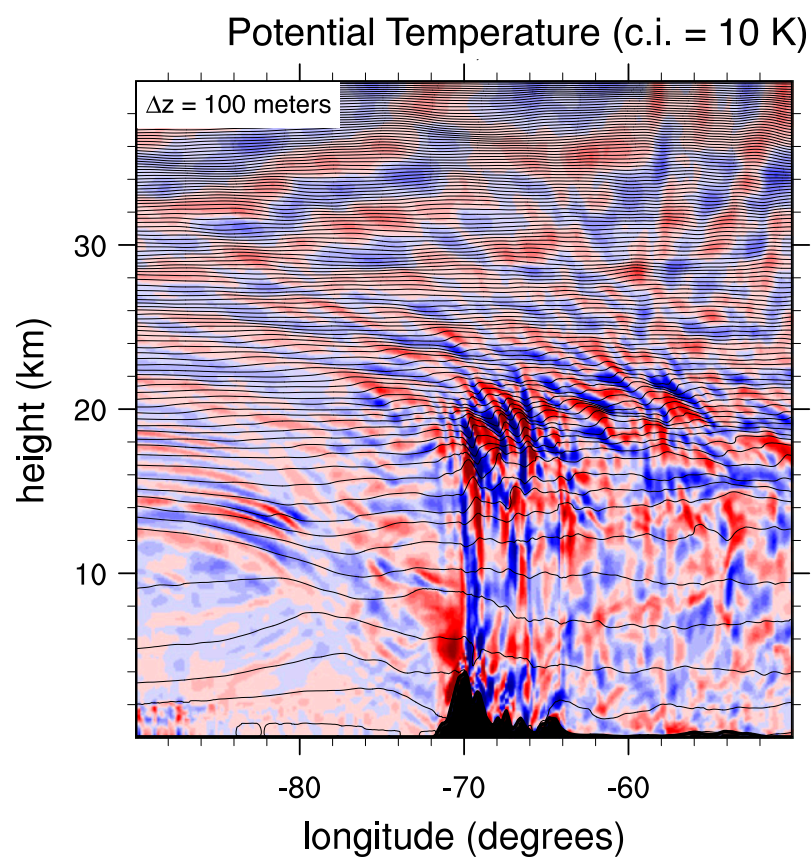

and Horizontal Divergence (fill, $\times 10^{4} \mathrm{~s}^{-1}$ )

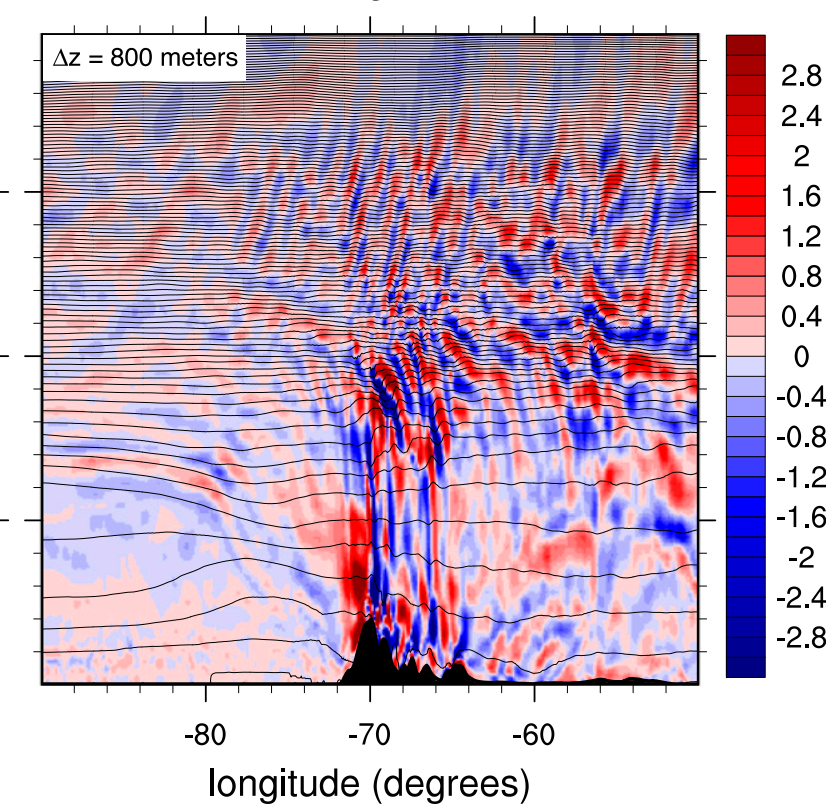

FIG. 4. Horizontal divergence and potential temperature in a longitudinal cross section in the Andes at $31^{\circ} \mathrm{S}$ latitude at forecast day 6 for the (left) $100-\mathrm{m}$ and (right) $800-\mathrm{m}$ simulations.

to the west of the mountains and waves above the tropopause east of the mountains. Spurious waves tilting to the east with increasing height are apparent in the $(15 \mathrm{~km}, 800 \mathrm{~m})$ solution above and to the east of the mountains in the stratosphere. The $(15 \mathrm{~km}, 400 \mathrm{~m})$ solution (not shown) still has the spurious waves but at reduced amplitude, and they are absent from the $(15 \mathrm{~km}$, $200 \mathrm{~m}$ ) solution (also not shown). The convergence of the flow fields for the mountain waves appears consistent with the convergence of the spectra.

A latitudinal cross section through a baroclinic wave ridge over the Southern Ocean for the $(15 \mathrm{~km}, 100 \mathrm{~m})$ simulation at day 5 is given in Fig. 5. The horizontal wind speed and the potential temperature reveal a strong midlatitude jet with strong vertical wind shear in the upper regions of the jet in addition to a surface front. Cross sections from the $(15 \mathrm{~km}, 100 \mathrm{~m})$ and $(15 \mathrm{~km}$, $800 \mathrm{~m}$ ) simulations, depicting the horizontal divergence and potential temperature fields, are shown in Fig. 6. In both solutions, there is strong horizontal divergence in the troposphere, where there is frontogenesis and latent heating, and also in the lower stratosphere. The striations in divergence in the stratosphere again arise from mesoscale inertia-gravity waves emitted by the synopticscale baroclinic wave and embedded in the upper portion of the jet [cf. with e.g., Fig. 3 of Plougonven and Snyder (2007)]. Differences between the two solutions are most apparent in the disappearance of the inertia-gravity waves in the $(15 \mathrm{~km}, 800 \mathrm{~m})$ simulation where the deep stratospheric waves are also much too strong. The weak stratospheric inertia-gravity waves tilting upward to the north are present in the $(15 \mathrm{~km}, 100 \mathrm{~m})$ solution. As in the case of the mountain waves, the $(15 \mathrm{~km}, 400 \mathrm{~m})$ and $(15 \mathrm{~km}, 200 \mathrm{~m})$ solutions (not presented) show convergence similar to the convergence in the spectra.

Figure 7 shows a longitudinal cross section at $2^{\circ} \mathrm{S}$ of horizontal divergence and potential temperature fields from the $(15 \mathrm{~km}, 100 \mathrm{~m})$ and $(15 \mathrm{~km}, 800 \mathrm{~m})$ simulations Indian Ocean-western Pacific region. There is a strong easterly jet with a horizontal extent greater than the

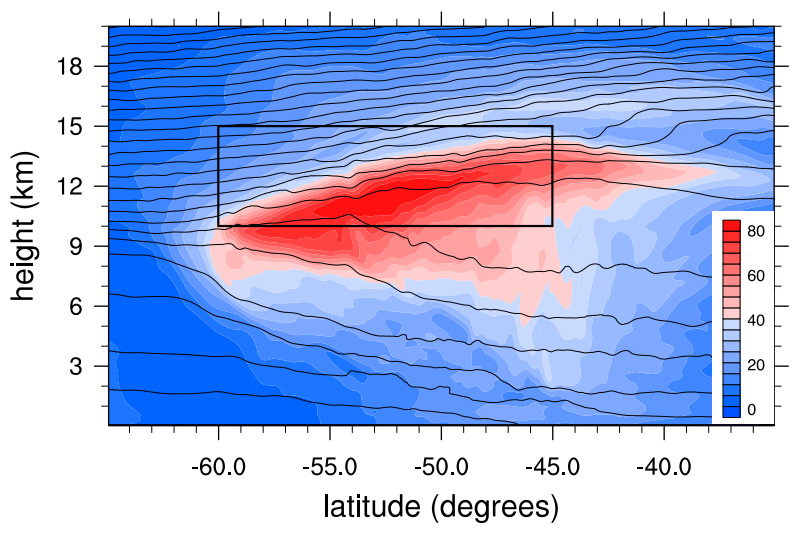

FIG. 5. Horizontal wind speed and potential temperature in a latitudinal cross section over the Southern Ocean at $65^{\circ} \mathrm{E}$ longitude at forecast day 5 for the $100-\mathrm{m}$ simulation. The box indicates the region shown in Fig. 10 


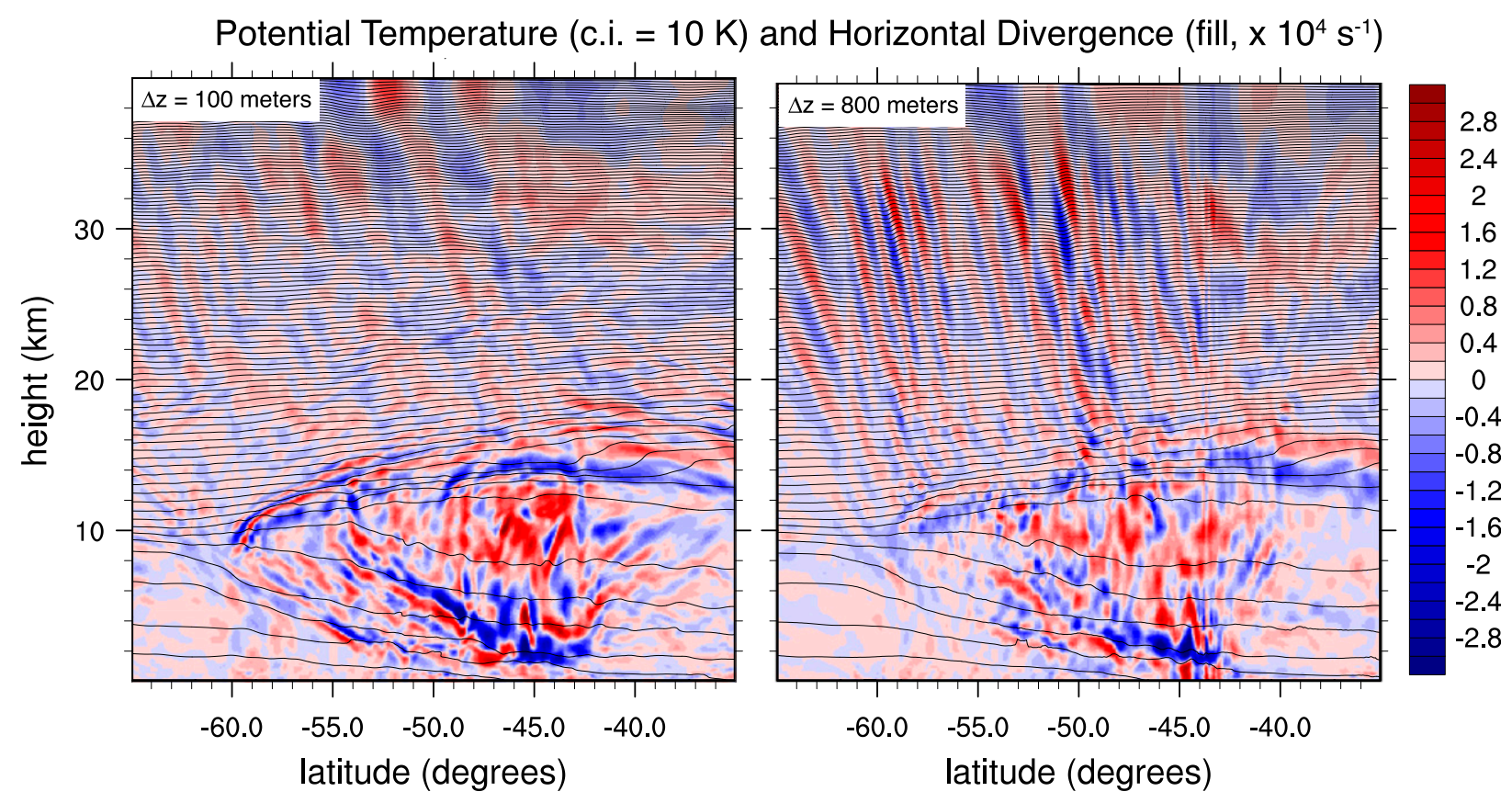

FIG. 6. Horizontal divergence and potential temperature in a latitudinal cross section over the Southern Ocean at $65^{\circ} \mathrm{E}$ longitude at forecast day 5 for the (left) 100-m and (right) 800-m simulations.

plotted domain, and a depth of a few kilometers, with maximum wind speeds at the tropopause. The jet is associated with two large counterrotating gyres. There is significant convective activity associated with the jet, and very strong stratification at the tropopause in both simulations. Above the tropopause, the $(15 \mathrm{~km}, 800 \mathrm{~m})$ solution depicts a coherent wave field with the waves tilted to the west with height. These waves are absent in the $(15 \mathrm{~km}, 100 \mathrm{~m})$ solution, where large-scale shallowly sloped waves are again much stronger in the $100-\mathrm{m}$
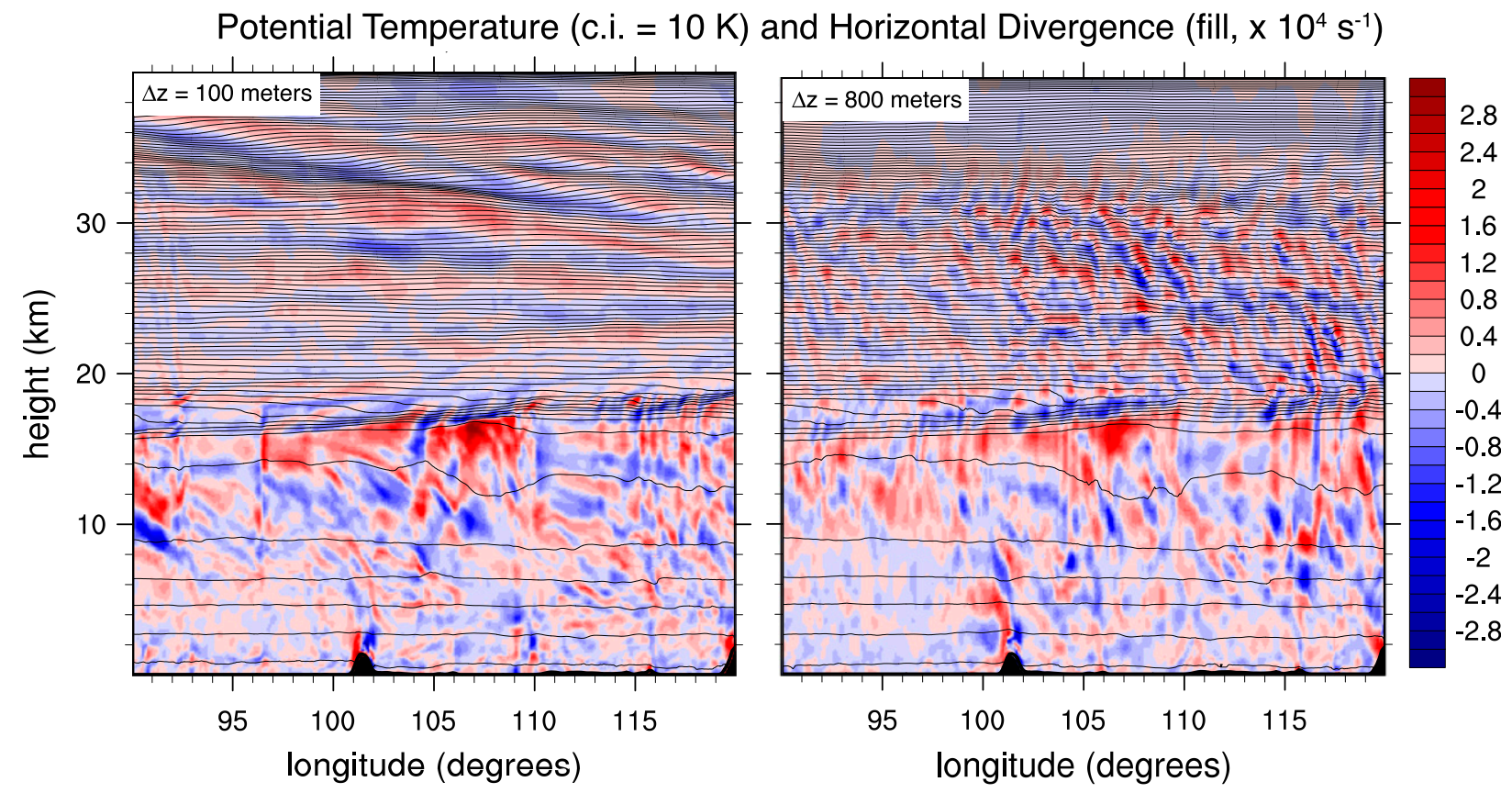

FIG. 7. Horizontal divergence and potential temperature in a longitudinal cross section in the Indian Ocean-western Pacific region at $2^{\circ} \mathrm{S}$ latitude at forecast day 5 for the (left) 100-m and (right) 800-m simulations. 
solution. The wave fields indicated by the horizontal divergence in the stratosphere in these simulations are completely different. The $(15 \mathrm{~km}, 400 \mathrm{~m})$ and $(15 \mathrm{~km}$, $200 \mathrm{~m}$ ) solutions show convergence similar to that with the mountain wave and baroclinic wave flow features we examined.

In the flow features shown in these three cross sections, all show increased noise and the appearance of spurious structures along with decreasing mesoscale kinetic energy with decreasing vertical resolution. However, it is often observed that noise in the solutions is accompanied by increases in kinetic energy in the higher wavenumbers of the spectrum (Skamarock 2004). In the MPAS results shown in Fig. 2, it appears that the noise and spurious structures do not contain significant energy, rather the MPAS spectra show decreased energy at the higher wavenumbers for the coarser vertical mesh results. The spurious stratospheric waves in Figs. 4, 6, and 7 have wavelengths $\leq 200 \mathrm{~km}$, where the model filters are actively damping them as indicated by the significant drop-off in the spectra shown in Fig. 2. Furthermore, the MPAS spectra decomposed in rotational and divergent components (not shown) behave as the full spectra with kinetic energy in the mesoscale region of the spectrum increasing with decreasing vertical mesh spacing. This is the case even for the divergent component of the spectra where we would look for energy from these modes. It appears that the resolved mesoscale structures and those at the resolution margin experience further scale collapse, both horizontally and vertically, with increasing vertical resolution, thus leading to increased mesoscale energy.

\section{Vertical resolution and model filters}

There are five processes in MPAS-A that directly result in kinetic energy dissipation: the second-order horizontal Smagorinsky filter, the background fourth-order horizontal filter, the gravity wave drag scheme, the boundary layer scheme, and dissipation in the vertical advection scheme applied to momentum. The KE dissipation associated with each of the processes is described in the appendix. Figure 8 shows the horizontally averaged kinetic energy dissipation in the $(15 \mathrm{~km}, 100 \mathrm{~m})$ meter simulation averaged over days 6 and 7. The dissipation is dominated by that occurring in the first few kilometers of the atmosphere (i.e., in the planetary boundary layer). Dissipation from the PBL scheme dominates the total dissipation in the troposphere and lower stratosphere, decaying slowly through the troposphere and more rapidly above the tropopause. The dissipation rates for the coarser vertical meshes (not shown) have similar distributions for the total dissipation but with less dissipation arising from the PBL scheme and increasing

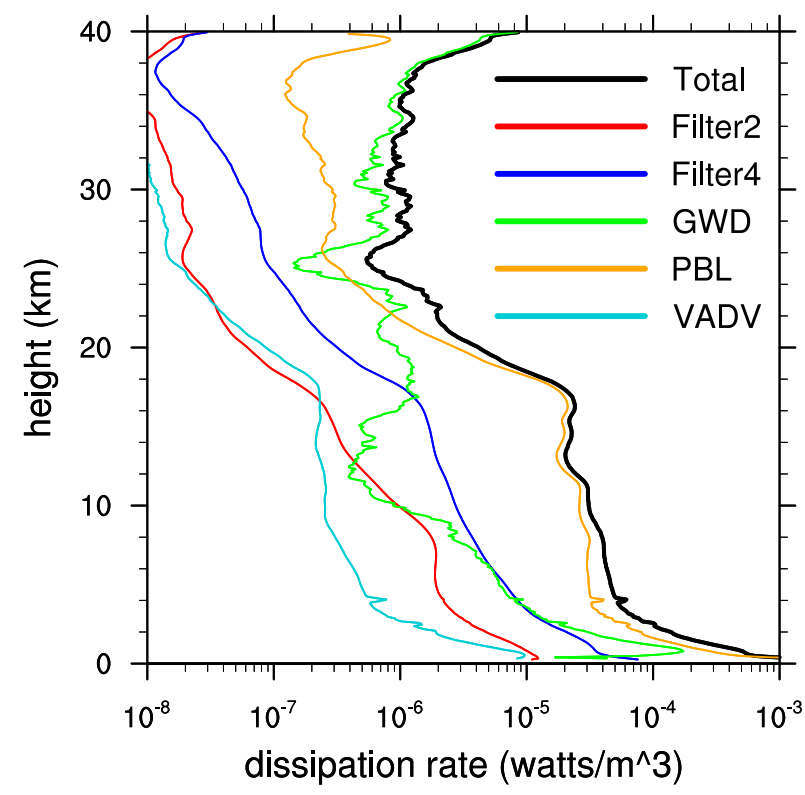

FIG. 8. Kinetic energy dissipation rate as a function of height in the $\Delta z=100$ - $\mathrm{m}$ simulation. The dissipation rate is averaged horizontally over days 6 and 7 .

dissipation from the vertical advection and gravity wave drag schemes. The dissipation rates also indicate that the $(15 \mathrm{~km}, 200 \mathrm{~m})$ solution is close to convergence. Throughout the troposphere and lower stratosphere, the PBL scheme always has the largest contribution to the dissipation in our simulations, consistent with the results found in other studies using different models and PBL schemes (e.g., Brune and Becker 2013; Malardel and Wedi 2016).

The YSU PBL scheme (Hong et al. 2006) used in MPAS employs a Richardson number-based formulation for computing the vertical eddy viscosity in the free atmosphere, and the eddy viscosity applied to momentum is given by

$$
K_{m}=\frac{l^{2} P_{r}}{\left(1+5 R_{i}\right)^{2}}\left|\frac{\partial U}{\partial z}\right|,
$$

where $R_{i}$ is the Richardson number, $P_{r}=\min \left(1+2.1 R_{i}, 4\right)$ is the Prandtl number, and $l$ is the length scale. In Hong et al. the reference length scale is $l=150 \mathrm{~m}$, but in the current MPAS formulation the length scale is $l=\max (\Delta z)$ $10,30 \mathrm{~m})$. The current formulation for the length scale was introduced by Hong (personal communication) based on WRF model forecast results. In the simulations presented here the length scales in the constant resolution regions of the vertical grids are $l=(80,40$, $30,30) \mathrm{m}$ for $\Delta z=(800,400,200,100) \mathrm{m}$, respectively.

The KE spectra in Fig. 2 shows increasing mesoscale $\mathrm{KE}$ with decreasing vertical mesh spacing in contrast to the idealized simulation results in Waite (2016, Fig. 11) and 


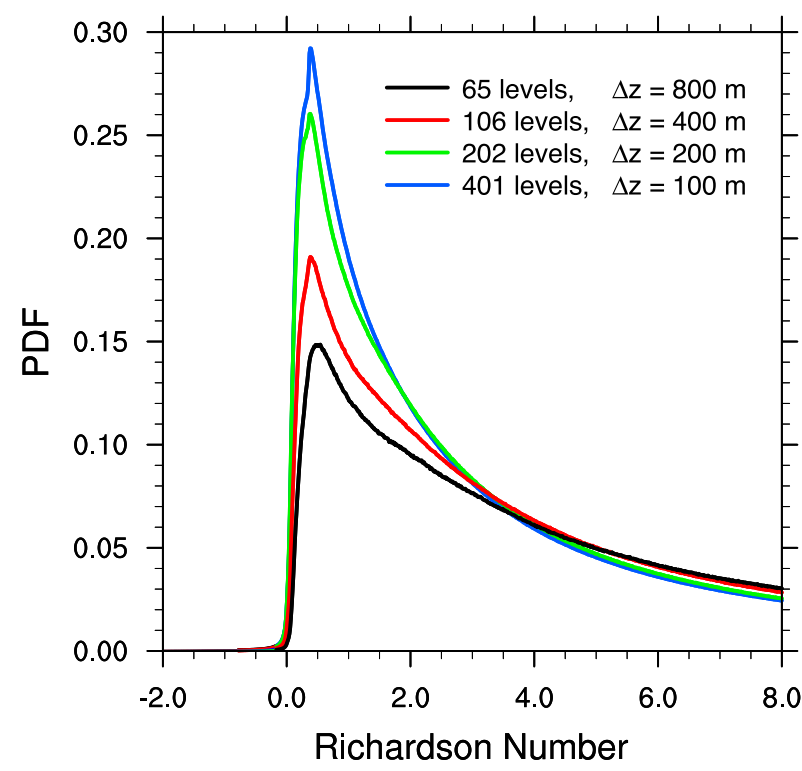

FIG. 9. Probability density function for the Richardson number at forecast day 5 for the $15-\mathrm{km}$ simulations.

Brune and Becker (2013, Fig. 1). One of the differences between our results and those of Waite (2016) is that the mixing length is fixed in the Waite formulation at $30 \mathrm{~m}$, whereas within the YSU scheme the mixing length increases with increasing vertical grid spacing. We have found significant sensitivity of the mesoscale KE to the length scale formulation in (6). Tests indicate that increasing (decreasing) the mixing length $l$ in (6) will decrease (increase) the mesoscale $\mathrm{KE}$, suggesting that the different mixing length formulations in the YSU scheme and in the Waite $R_{i}$-based scheme are responsible for the different mesoscale spectra convergence behaviors. We also find that decreasing the mixing length also increases visible noise and the amplitude of spurious structures, so tuning the PBL scheme formulation based solely on the mesoscale KE spectrum may lead to degraded results for vertically underresolved configurations.

Vertical mixing in the PBL scheme occurs with low Richardson numbers, and Waite used this as a measure of convergence [see Waite (2016), Fig. 12]. Figure 9 shows the probability density functions (PDF) for the Richardson number in the $15-\mathrm{km}$ simulations. Consistent with Waite, we find convergence of the PDF as vertical mesh spacing is reduced. In both cases it is characterized by the convergence of an increasing peak PDF amplitude with decreasing vertical mesh spacing, consistent with the mesoscale spectra convergence.

Figure 5 shows a cross section of the horizontal wind speed and potential temperature fields from the $(15 \mathrm{~km}, 100 \mathrm{~m})$ simulation through a ridge in a baroclinic wave in the southern Indian Ocean at forecast day 5,

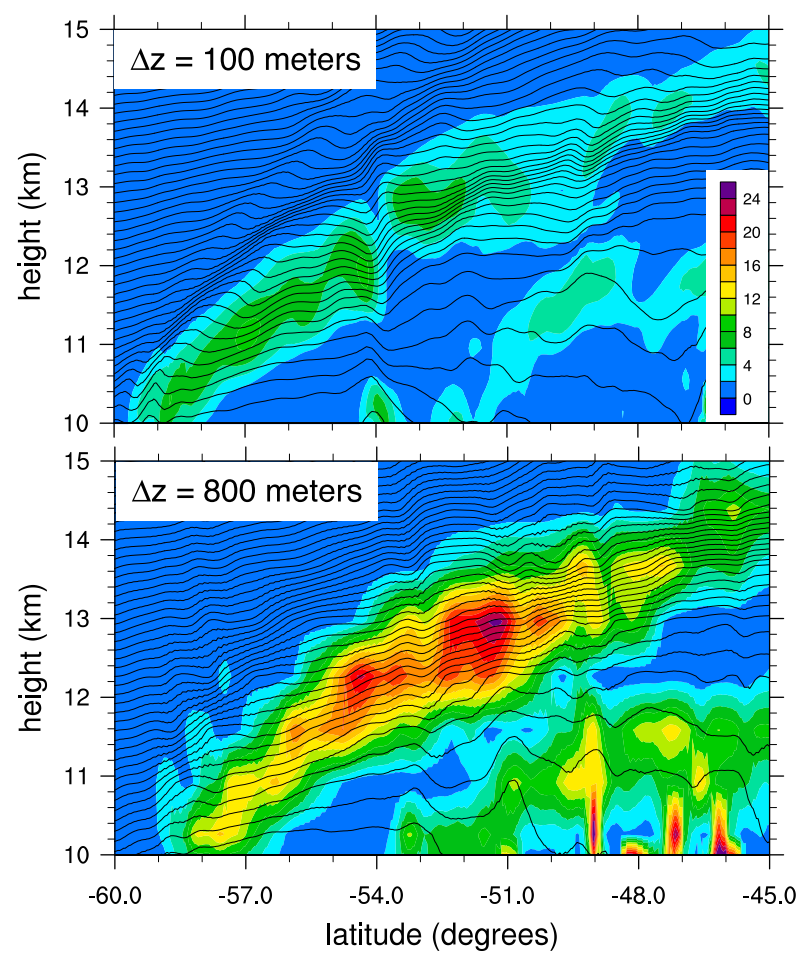

FIG. 10. Vertical eddy viscosity $\left(\mathrm{m}^{2} \mathrm{~s}^{-1}\right.$, color) and potential temperature (contour interval $=2 \mathrm{~K}$ ) in a latitudinal cross section over the Southern Ocean at $65^{\circ} \mathrm{E}$ longitude at forecast day 5 for the (top) $\Delta z=100-\mathrm{m}$ and (bottom) $\Delta z=800-\mathrm{m}$ simulations.

with a box highlighting the region of strong vertical wind shear at the upper edge of the jet. Figure 10 shows vertical cross sections of the vertical eddy viscosity and potential temperature fields from the $(15 \mathrm{~km}, 100 \mathrm{~m})$ and $(15 \mathrm{~km}, 800 \mathrm{~m})$ simulations through a ridge in the box from Fig. 5. In the converged $(15 \mathrm{~km}, 100 \mathrm{~m})$ solution the eddy viscosities are significantly smaller than those from the $(15 \mathrm{~km}, 800 \mathrm{~m})$ solution. The upper edge of the jet for the $(15 \mathrm{~km}, 800 \mathrm{~m})$ simulation shows distinct stairsteps along its edge in both the eddy viscosity and the potential temperature. This stair-step structure is very similar to those shown in past studies such as Pecnick and Keyser (1989), Persson and Warner (1991), etc.

\section{Convergence for other horizontal mesh spacings}

\section{a. Coarsening the horizontal mesh}

We have performed the same set of vertical resolution experiments using horizontal meshes with 120-, 60-, and $30-\mathrm{km}$ cell spacing. The KE spectra for these experiments (not shown) have convergence characteristics similar to the $15-\mathrm{km}$ mesh results although slightly less pronounced (i.e., $\Delta z \leq 200 \mathrm{~m}$ is needed before the spectra are converged). The lack of KE spectra convergence appears 


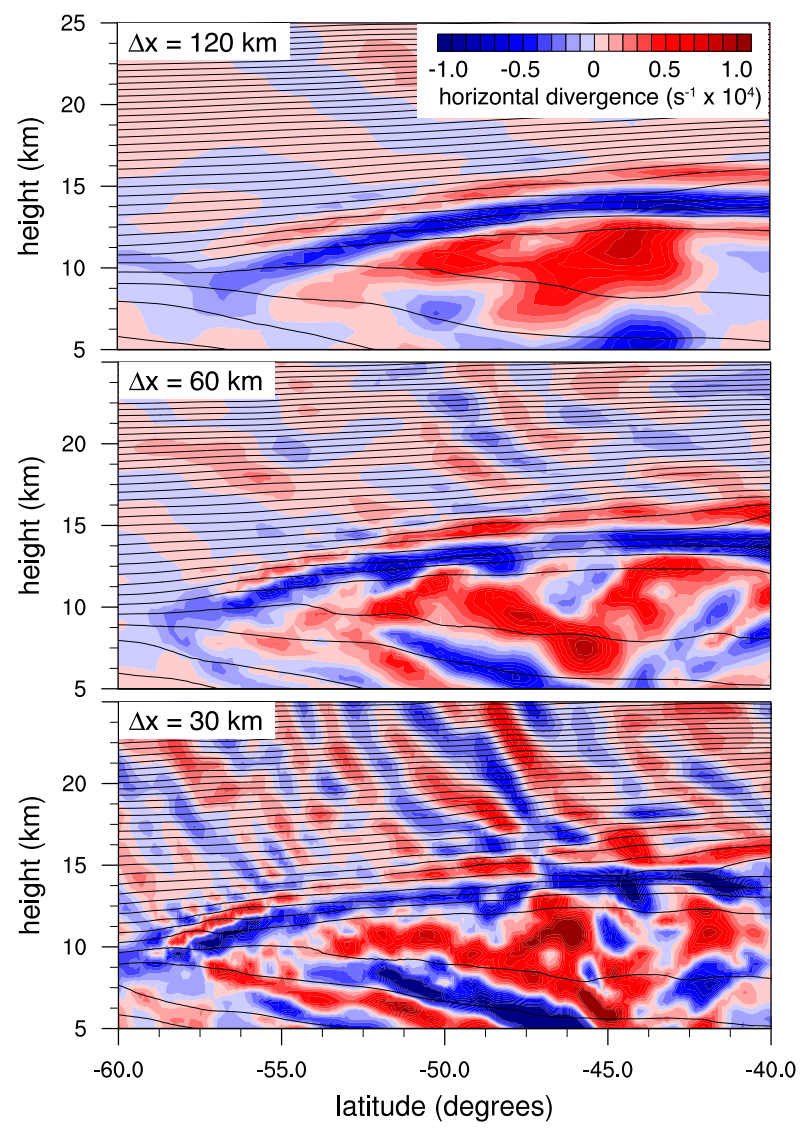

FIG. 11. Vertical cross sections of horizontal divergence at $65^{\circ} \mathrm{E}$ longitude at forecast day 5 from simulations with $\Delta z=800 \mathrm{~m}$ and 120-, 60-, and 30-km horizontal cell spacing meshes.

most prominently in the divergent component of the KE spectra. In contrast, at coarser horizontal resolutions, the spurious flow features apparent in the $(15 \mathrm{~km}, 800 \mathrm{~m})$ solution become less apparent and are almost absent in the $(120 \mathrm{~km}, 800 \mathrm{~m})$ solution as shown in Fig. 11 that depicts the vertical cross sections for the $\Delta z=800-\mathrm{m}$ solutions through the Southern Hemisphere ridge. These coarser-horizontal-mesh solutions can be compared with the cross sections for the $(15 \mathrm{~km}, 800 \mathrm{~m})$ results in Fig. 6; the attenuation of the spurious structures with coarser horizontal resolution is apparent. These results indicate that $\mathrm{KE}$ dissipation from the $\mathrm{PBL}$ scheme strongly influences the convergence of the $\mathrm{KE}$ spectra in these simulations but has less influence on the convergence of the flow structures. Importantly, the KE spectra convergence as a function of vertical resolution is not significantly influenced by the horizontal resolution.

\section{b. Convection-permitting horizontal mesh results}

We have performed experiments using a $15-3-\mathrm{km}$ variable-cell spacing mesh depicted in Fig. 12, where the

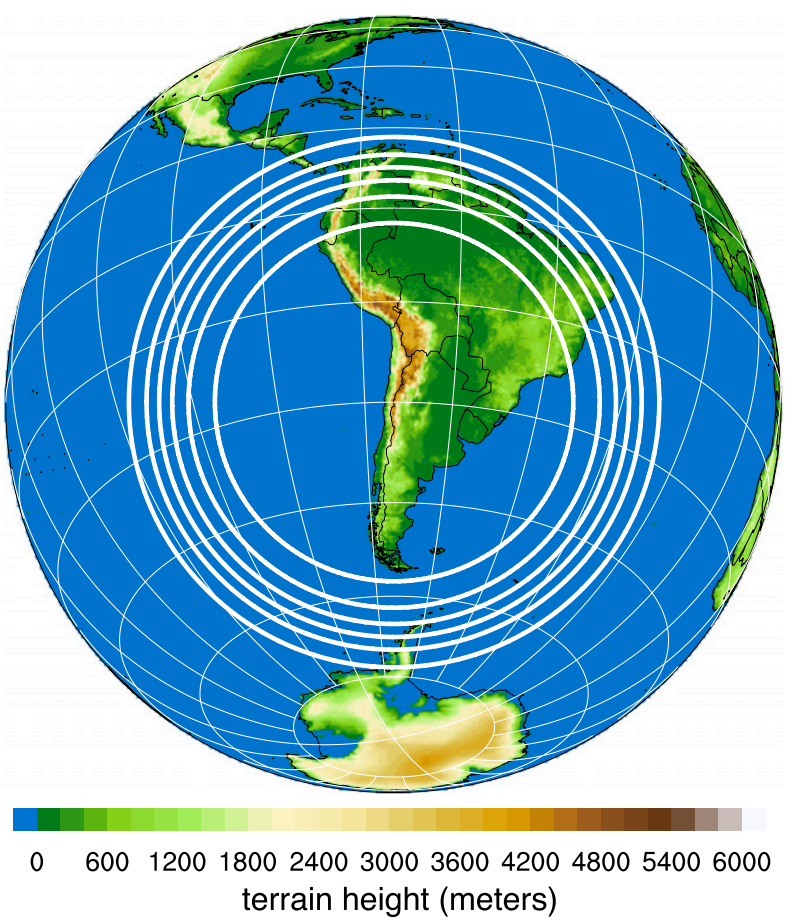

FIG. 12. MPAS 15-3-km mesh and terrain with refinement over the Andes. Cell spacing is contoured in white with an interval of $2 \mathrm{~km}$ starting at $4 \mathrm{~km}$.

$3-\mathrm{km}$ high-resolution region is placed over the Andes in South America. Cross sections for the (15-3 km, $800 \mathrm{~m})$ and $(15-3 \mathrm{~km}, 100 \mathrm{~m})$ solutions at forecast day 6 are presented in Fig. 13, and these can be compared with the cross sections from the $15-\mathrm{km}$ horizontal mesh simulations given in Fig. 4. (Note that the color scale for horizontal divergence differs by an order of magnitude between the two plots.) The $(15-3 \mathrm{~km}, 800 \mathrm{~m})$ meter solution shows spurious waves above the Andes similar to those in the $(15 \mathrm{~km}, 800 \mathrm{~m})$ solution, and these waves are absent from the $(15-3 \mathrm{~km}, 100 \mathrm{~m})$ and $(15 \mathrm{~km}, 100 \mathrm{~m})$ solutions. Some of the gravity waves appearing above the mountains to the east in the $(15-3 \mathrm{~km}, 100 \mathrm{~m})$ meter solution are not resolved in the other solutions because the horizontal wavelengths of these waves is of order 6-10 $\Delta x$ in the region just above the tropopause (between 66 and $68 \mathrm{~W}$ and $20-25 \mathrm{~km}$ in height) in this $3-\mathrm{km}$ mesh-spacing region of the $(15-3 \mathrm{~km}, 100 \mathrm{~m})$ simulation. The $(15-3 \mathrm{~km}, 100 \mathrm{~m})$ solution also exhibits shallowly sloped layers of alternating high or low stability in stratosphere that appear to be inertia-gravity waves. These waves are much weaker at lower vertical resolution.

Figure 14 show vertical cross sections through the Southern Hemisphere ridge examined earlier in the $15-\mathrm{km}$ simulation results, but with the simulations here using the 15-3-km mesh with the high-resolution region placed over the ridge. These results can be compared 


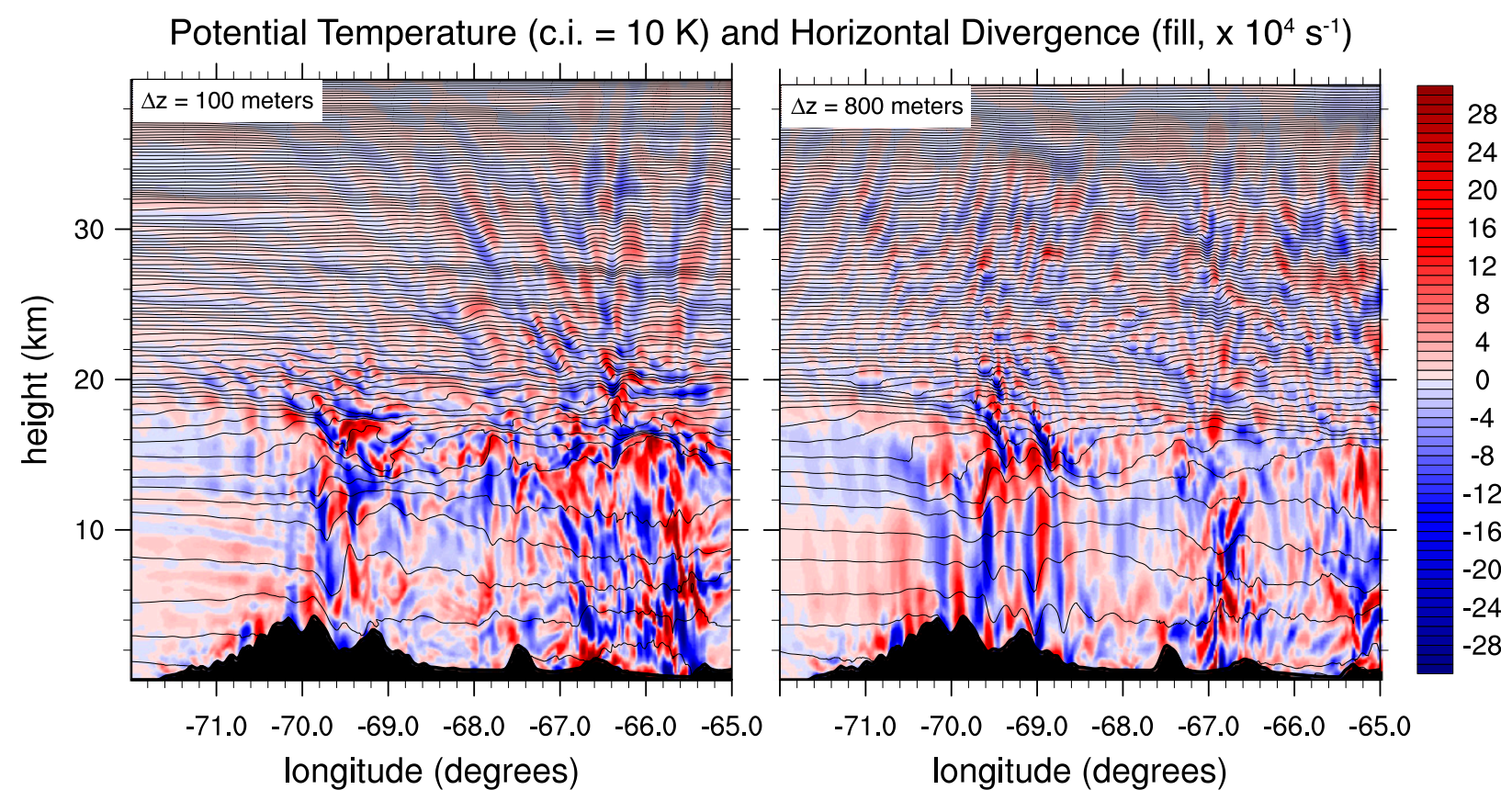

FIG. 13. Vertical cross sections through the Southern Andes for the (left) $\Delta z=100$-m and (right) $\Delta z=800-\mathrm{m} 15-3$-km simulations where the high-resolution region is centered on the Andes.

with those presented in Fig. 6 for the 15-km simulations. The horizontal divergence field for the $(15-3 \mathrm{~km}, 800 \mathrm{~m})$ result shows poorly resolved unphysical structures in the divergence within the jet, and these spurious structures are much more pronounced compared to those in the
$(15 \mathrm{~km}, 800 \mathrm{~m})$ simulation. This is the region of the jet where the stability is low, and it appears that the atmosphere is attempting to overturn on resolved scales. Also apparent is increased amplitude in the spurious stratospheric waves above the jet (waves tilting the south with

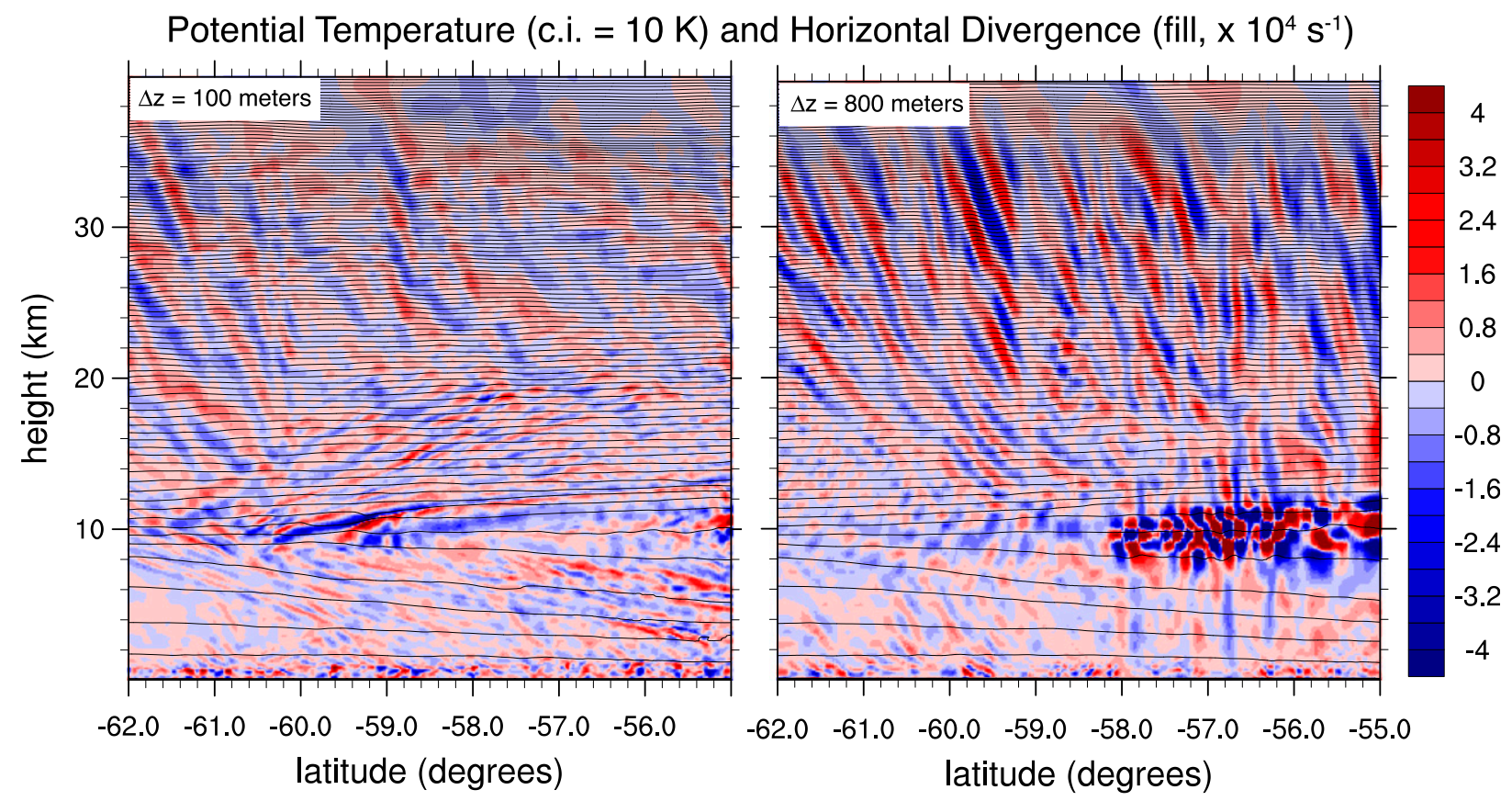

FIG. 14. Vertical cross sections through the ridge in the Southern Hemisphere baroclinic wave for the (left) $\Delta z=100-\mathrm{m}$ and (right) $\Delta z=$ 800-m 15-3-km simulations where the high-resolution region is centered on the ridge. 


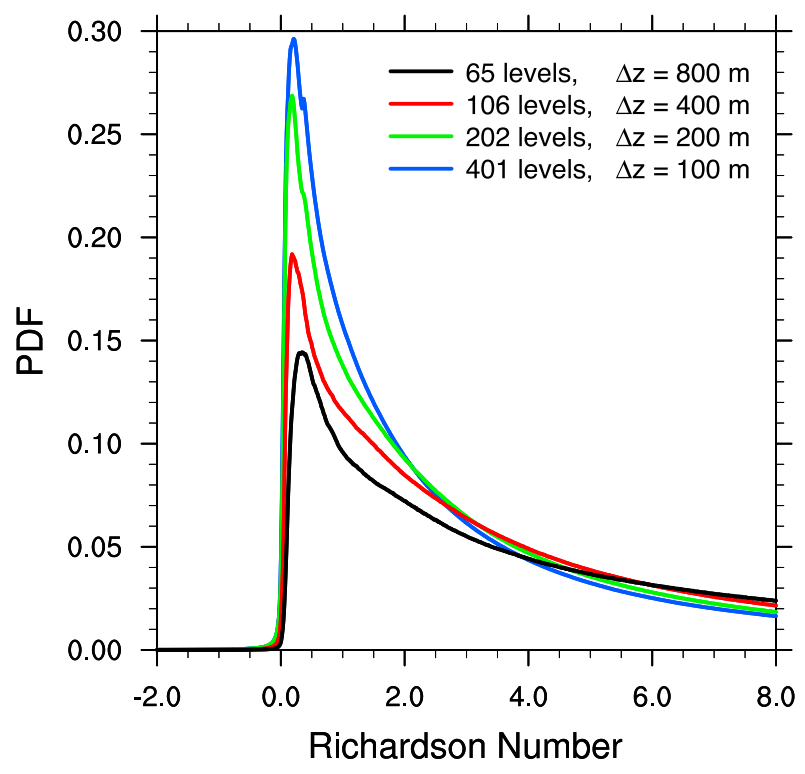

FIG. 15. Probability density function of the Richardson number for the $15-3-\mathrm{km}$ simulations at day 5 , sampled from the regions where $\Delta x \leq 4 \mathrm{~km}$.

height). The $(15-3 \mathrm{~km}, 100 \mathrm{~m})$ meter solution appears fairly well resolved, especially in the upper region at the southern edge of the jet.

The Richardson number PDFs for the 15-3-km simulations, given in Fig. 15, are computed from simulations with the high-resolution region centered over the Andes (see Fig. 12), the Southern Hemisphere ridge, and a region of active convection in the Maritime continents region. The PDFs are computed using results from the regions where $\Delta x \leq 4 \mathrm{~km}$. Compared with the PDFs from the $15-\mathrm{km}$ results (Fig. 9), the high-resolution results are not quite as well converged at $\Delta z=200 \mathrm{~m}$. While the peak values for the $\Delta z=100$ - and $\Delta z=200-\mathrm{m}$ simulations are similar in the $15-\mathrm{km}$ and $15-3-\mathrm{km}$ results, the differences between the PDFs in the $\Delta z=100$ - and 200-m solutions for $\sim 0.5 \leq R_{i} \leq 2$ is significantly more pronounced in the $15-3-\mathrm{km}$ results. This region is where the Richardson number weighting in (6) for the vertical eddy viscosity is falling off rapidly. The Richardson numbers for the peak values are also slightly shifted, from approximately 0.4 in the $15-\mathrm{km}$ PDF to approximately 0.2 in the $3-\mathrm{km}$ PDF. The Richardson number peak of approximately 0.2 implies vertical shearing instability, but the eddies and breaking waves do not explicitly appear because the filters remove them.

\section{Summary and discussion}

Using MPAS-A with full-physics NWP configurations, we have examined solution convergence in the free atmosphere as a function of vertical resolution. Specifically, we have produced forecasts where the vertical mesh spacing is limited to $800,400,200$, and $100 \mathrm{~m}$ on horizontal meshes with cell spacings of $120,60,30,15$, and $15-3 \mathrm{~km}$. Three measures of convergence are examined: kinetic energy spectra, flow features, and Richardson number PDFs. For the $15-\mathrm{km}$ horizontal mesh (nominally the current operational global NWP resolution), convergence in all three measures occurs at approximately $\Delta z \leq 200 \mathrm{~m}$. Nonconvergence at coarser vertical mesh spacings is accompanied by noise and spurious flow features, reduced levels of $\mathrm{KE}$ in the mesoscale region of the spectra, and reduced Richardson number frequencies in the low $R_{i}$ region of the $R_{i}$ PDFs.

Compared to the $15-\mathrm{km}$ solutions, coarser horizontal mesh solutions show similar convergence for the KE spectra, but show less noise and fewer spurious flow features at coarser vertical resolutions compared to the higher horizontal resolution mesh results. Convectionpermitting mesh results, produced using a variableresolution $15-3-\mathrm{km}$ mesh with the high-resolution region centered on convergence-critical flow features identified in the $15-\mathrm{km}$ solutions, exhibit signs of convergence at $\Delta z=100 \mathrm{~m}$. The $R_{i}$ number PDFs for the 15-3-km simulations show convergence similar to the $15-\mathrm{km}$ solutions with slightly more variation between the $\Delta z=200$ - and 100-m solution PDFs. These measures are less definitive than those examined in simulations using other horizontal mesh spacings because we do not have spectra for the variable-resolution simulations.

Vertical scale collapse can be limited by vertical filters and by horizontal filters in the case of sloping features where the horizontal and vertical wavenumbers are proportional to each other. These filters also dissipate kinetic energy. In the free atmosphere, the Richardson numberbased vertical mixing in the YSU PBL scheme is the dominant kinetic energy sink in the simulations. Even though the vertical eddy viscosities diagnosed in the PBL scheme are larger for the coarser vertical meshes (in part due to the larger length scales used in the eddy viscosity calculation), the larger eddy viscosities are not sufficient to produce smooth well-resolved solutions.

Overall, the results from this study largely confirm and further illuminate those of past studies. We observe noise generated at sloping fronts when the vertical mesh spacing is too coarse, similar to that found in other studies (e.g., Pecnick and Keyser 1989). Flow features and $\mathrm{KE}$ spectra converge consistently in the $15-\mathrm{km}$ horizontal mesh results, and this convergence is reached when the mesh aspect ratio $\Delta z / \Delta x \sim 1 / 100$ [i.e., similar to the frontal slope arguments from Pecnick and Keyser in (2)]. On coarser horizontal meshes the noise is reduced 
or absent for the coarser vertical resolutions, again consistent with the frontal-slope argument in (2).

Pecnick and Keyser (1989) and other studies in this vein (e.g., Persson and Warner 1991; Snyder et al. 1993; Lean and Clark 2003; Iga et al. 2007) focused on the accurate discrete representation of resolvable flow features, but our results show that the convergence of flow features does not guarantee convergence in other metrics, specifically in the KE spectrum and in the $R_{i} \mathrm{PDF}$. Our results are largely consistent with Waite (2016) who looked at convergence of the KE spectrum and the $R_{i} \mathrm{PDF}$. Both studies suggest that a vertical mesh spacing $\Delta z \leq 200 \mathrm{~m}$ is needed to reach approximate convergence for both measures, and these results are consistent with the result reported by Cullen (2017) who looked at Froude number convergence in real-data forecasts using the Unified Model. The study by Waite used an idealized baroclinic jet that had a mesoscale spectrum much shallower than the observed $k^{-5 / 3}$ slope, and the study by Cullen used a horizontal mesh with $\Delta x \approx 40 \mathrm{~km}$ where the mesoscale portion of the spectrum is not resolved. Thus the results presented here extend some of the results from these previous studies. Both Waite and Cullen argue that their results provide evidence for stratified turbulence playing an important role in dynamics of the atmosphere, and Waite argues that stratified turbulence may be an explanation for the character of the mesoscale spectrum. The results of this study may further support a stratified turbulence interpretation of the mesoscale dynamics, but addressing that question is beyond the scope of this paper.

Our results also show that part of the solution sensitivity to vertical resolution comes from mesoscale inertia-gravity waves that are well resolved horizontally but poorly resolved vertically except at the highest vertical resolutions. In each of the example cross sections presented here, much stronger inertia-gravity waves (revealed as shallowly sloping striations in horizontal divergence and static stability) are present in the stratosphere at $\Delta z=100 \mathrm{~m}$ than at $\Delta z=$ $800 \mathrm{~m}$. The importance of inertia-gravity waves to the mesoscale kinetic energy spectrum in the stratosphere is consistent with the idealized simulations of Waite and Snyder (2009) and the analysis of Burgess et al. (2013) of highresolution global forecasts. It is also supported by observational estimates of the intrinsic-frequency spectrum for horizontal velocity (Hertzog et al. 2002), which show a near-inertial peak in the high-latitude stratosphere.

Spurious flow features and noise can contaminate solutions in research and operational applications, and the results presented herein suggest that vertical mesh spacings with $\Delta z \leq 200 \mathrm{~m}$ should be used to achieve convergence with mesoscale and higher horizontal resolution global and regional atmospheric model applications. Most model applications use much coarser vertical meshes in the free atmosphere, for example the MPAS model used in this study has a default vertical mesh spacing approaching $800 \mathrm{~m}$ at the tropopause, the NCEP GFS configuration is similarly coarse and the Met Office Unified Model configuration has a vertical mesh spacing over $500 \mathrm{~m}$ at the midlatitude tropopause. In contrast, the ECMWF IFS configuration has a vertical mesh spacing of approximately $300 \mathrm{~m}$ throughout the troposphere. This vertical mesh spacing may well be producing near-converged solutions for the horizontal truncation in the IFS (currently T1279), although solution convergence studies did not drive this aspect of the IFS configuration (Richard Forbes, ECMWF, personal communication). The IFS experience suggests that consistent horizontal-vertical resolution benefits analysis and forecast accuracy. Both the Unified Model and the GFS will soon upgrade to higher vertical resolution, so it appears that the operational models are evolving to more consistent horizontal-vertical resolution. Practically speaking, the cost of increasing vertical resolution is generally less than the cost of increasing horizontal resolution, so higher vertical-resolution bears examination along with increasing horizontal resolution and/or including more costly model physics in an application's configuration.

For coarser-mesh configurations, for example those used in ensembles, subseasonal to seasonal forecast, or in climate studies, it appears that spurious flow features and noise are adequately filtered at coarser vertical resolutions. The KE spectra did not show convergence until $\Delta z \leq 200 \mathrm{~m}$ in our results, but it is possible that the PBL scheme could be tuned to result in more-closely converged $\mathrm{KE}$ spectra at the coarser vertical resolutions. Determining optimal configurations for applications is not the focus of this paper, and optimal configurations are likely to be somewhat dependent on model numerics and filters, and model physics, particularly the PBL scheme.

Acknowledgments. Computing support for this work was provided by NCAR's Computational and Information Systems Laboratory. The National Center for Atmospheric Research is supported by the National Science Foundation. The authors would also like to acknowledge the two anonymous reviewers for helping to improve the clarity of this manuscript.

\section{APPENDIX}

\section{Kinetic Energy Dissipation in MPAS}

There are five processes that serve to remove kinetic energy in the simulated flow in MPAS. Three of these processes are part of the MPAS fluid-flow solver: the horizontal Smagorinsky mixing, the horizontal fourth-order 
filter, and dissipation in the vertical advection scheme (MPAS uses the vector-invariant form of the horizontal momentum equations, and there is no dissipation associated with the horizontal discretization of these terms outside of the explicit filters). Additional dissipation occurs in the model physics, specifically the boundary layer scheme (that mixes throughout atmosphere) and the gravity wave drag scheme. The equation for the kinetic energy is derived by taking the dot product of the horizontal momentum equation with the horizontal velocity. We examine each KE-dissipative process in MPAS and determine the KE dissipation and KE transport associated with them.

\section{a. Horizontal Smagorinsky mixing}

MPAS uses a vector-invariant form of the horizontal momentum equations, and the corresponding secondorder dissipation is written using the vertical vorticity and horizontal divergence, here in vector form:

$$
\frac{\partial V}{\partial t}=\cdots+K_{2}(\nabla D-\nabla \times \zeta),
$$

where $D$ is the horizontal divergence and $\zeta$ is the relative vertical vorticity. The formulation (A1) is not cast in conservative form as analyzed in Klemp (2017) and written as

$$
\frac{\partial V}{\partial t}=\cdots+\nabla\left(K_{2} \mathrm{D}\right)-\nabla \times\left(K_{2} \zeta\right)
$$

so the formulation (A1) should be viewed as a nonconservative numerical filter. The conservative formulation (A2) will be in the next MPAS release.

To form the KE equation with the MPAS Smagorinsky filter (A1), we take the dot product of $V$ with (A1) and, with some algebraic manipulations, the result can expressed as

$\frac{\partial}{\partial t}\left(\frac{V^{2}}{2}\right)=\cdots+K_{2} \nabla \cdot(V D+V \times \zeta)-K_{2}\left(D^{2}+\zeta^{2}\right)$,

where the first term on the rhs of (A3) is the turbulent transport and $-K_{2}\left(D^{2}+\zeta^{2}\right)$ is the energy dissipation. Casting the energy dissipation terms in (A3) in Cartesian coordinates

$$
\begin{aligned}
D^{2}+\zeta^{2}= & \left(\frac{\partial u}{\partial x}\right)^{2}+\left(\frac{\partial u}{\partial y}\right)^{2}+\left(\frac{\partial v}{\partial x}\right)^{2}+\left(\frac{\partial v}{\partial y}\right)^{2} \\
& +2\left(\frac{\partial u}{\partial x} \frac{\partial v}{\partial y}-\frac{\partial v}{\partial x} \frac{\partial u}{\partial y}\right)
\end{aligned}
$$

Note that the rightmost term in (A4), $2\left(u_{x} v_{y}-v_{x} u_{y}\right)$, does not appear in second-order mixing formulations where the Laplacian is cast directly in terms of the velocities (e.g., Gill 1982, section 4.6). The additional term, however, will integrate to zero over the globe, hence the two forms produce the same net global dissipation. The differences reveal the nonuniqueness inherent in decomposing the KE dissipation operator into turbulent fluxes and local KE dissipation.

\section{b. Fourth-order horizontal filter}

The fourth-order background filter in MPAS is computed by applying the second-order operator twice [i.e., $\left.\nabla^{2}\left(\nabla^{2} V\right)\right]$, as opposed to the applying a fourth-order filter in each coordinate directly $\nabla^{4} V$ as often done in atmospheric models. Using the MPAS vector-invariant form of the dissipation operator, we can cast the horizontal velocity equation with the fourth-order filter term as

$$
\frac{\partial V}{\partial t}=\cdots-\left\{K_{D 4} \nabla(\nabla \cdot \nabla D)+K_{\zeta 4} \nabla \times[\nabla \times(\nabla \times \zeta)]\right\},
$$

where $K_{D 4}$ and $K_{\zeta 4}$ are the hyperviscosities with units $\mathrm{m}^{4} \mathrm{~s}^{-1}$. In the MPAS formulation, the two hyperviscosities allow for different levels of filtering associated with the horizontally divergent modes and the vertical vorticity modes in the horizontal velocity fields.

Forming the KE equation by taking the dot product of (A5) with the velocity, we can write the KE dissipation terms as

$$
\begin{aligned}
\frac{\partial}{\partial t}\left(\frac{V^{2}}{2}\right)=\cdots & -K_{D 4} \nabla \cdot\left(D \nabla D-V \nabla^{2} D\right) \\
& +K_{\zeta 4} \nabla \cdot[V \times \nabla \times(\nabla \times \zeta)+\zeta \times \nabla \times \zeta] \\
& -K_{D 4}(\nabla D)^{2}-K_{\zeta 4}(\nabla \times \zeta)^{2},
\end{aligned}
$$

where the first two terms on the rhs of (A6) represents turbulent transport that redistributes the $\mathrm{KE}$ and the final two terms are the KE dissipation.

\section{c. Gravity wave drag}

The gravity wave drag scheme can be represented as a source term to the horizontal velocity, and the $\mathrm{KE}$ equation is

$$
\frac{\partial}{\partial t}\left(\frac{V^{2}}{2}\right)=\cdots+V \cdot \Delta V_{\mathrm{gwd}},
$$

where $\Delta V_{\mathrm{gwd}}$ is the gravity wave drag forcing, and the $\mathrm{KE}$ dissipation is the rhs term $V \cdot \Delta V_{\text {gwd }}$. 


\section{d. Planetary boundary layer scheme}

The YSU planetary boundary layer scheme in MPAS (Hong et al. 2006, MWR) applies second-order local vertical mixing to the horizontal momentum throughout the depth of the atmosphere. Within the PBL nonlocal mixing is also applied. Our interest at present is the dissipation in the free atmosphere, hence we consider only the local vertical mixing. The vertical dissipation term has the following form:

$$
\frac{\partial V}{\partial t}=\cdots+\frac{\partial}{\partial z}\left(K_{m} \frac{\partial V}{\partial z}\right)
$$

The horizontal kinetic energy equation terms associated with the PBL mixing from the local scheme are

$$
\frac{\partial}{\partial t}\left(\frac{V^{2}}{2}\right)=\cdots+\frac{\partial}{\partial z}\left[K_{m} \frac{\partial}{\partial z}\left(\frac{V^{2}}{2}\right)\right]-K_{m}\left(\frac{\partial V}{\partial z}\right)^{2},
$$

where $K_{m}$ is the eddy viscosity from the PBL scheme and has units $\mathrm{m}^{2} \mathrm{~s}^{-1}$. As before, the first term on the RHS is a vertical transport of horizontal kinetic energy and the second term is the dissipation of kinetic energy.

The vertical eddy viscosity is a function of the Richardson number (for a dry atmosphere, see Hong et al. (2006) for the moist atmosphere equivalent):

$$
R_{i}=\frac{g}{\theta} \frac{\partial \theta}{\partial z} /\left(\frac{\partial V}{\partial z}\right)^{2} .
$$

In a stable atmosphere, and here for simplicity ignoring the effects of moisture, the eddy viscosity is calculated as

$$
K_{m}=\frac{P_{r} \times l^{2}}{\left(1+5 R_{i}\right)^{2}} \times\left|\frac{\partial \mathbf{V}}{\partial z}\right|,
$$

where the Prandtl number $P_{r}=1+2.1 R_{i}$, and it is bounded $1<P_{r}<4$. The mixing length $l=\Delta z / 10$ with a fixed lower bound of $30 \mathrm{~m}$ (in the current WRF Version 4 and MPAS Version 5.1 releases).

\section{e. Vertical advection}

MPAS uses a third-order upwinded transport scheme for vertical advection of the horizontal velocity. The third-order scheme is equivalent to a fourth-order centered scheme plus a fourth-order filter with a hyperviscosity proportional to the vertical mesh spacing and the vertical velocity. Specifically, the filter term in the horizontal momentum equation can be written as

$$
\frac{\partial V}{\partial t}=\cdots-\frac{\partial}{\partial z}\left(K_{4} \frac{\partial^{3} V}{\partial z^{3}}\right)
$$

where the hyperviscosity $K_{4}=w \Delta z^{3} / 12$. The KE equation associated with the vertical advection is

$$
\frac{\partial}{\partial t}\left(\frac{V^{2}}{2}\right)=\cdots-\left[K_{4}\left(V V_{z z z}-V_{z} V_{z z}\right)\right]_{z}-K_{4} V_{z z}^{2},
$$

where the first term on the rhs of (11) is a turbulent transport and the second term is the KE dissipation.

\section{REFERENCES}

Billant, P., and J.-M. Chomaz, 2001: Self-similarity of strongly stratified inviscid flows. Phys. Fluids, 13, 1645-1651, https:// doi.org/10.1063/1.1369125.

Brune, S., and E. Becker, 2013: Indications of stratified turbulence in a mechanistic GCM. J. Atmos. Sci., 70, 231-247, https:// doi.org/10.1175/JAS-D-12-025.1.

Burgess, B. H., A. R. Erler, and T. G. Shepherd, 2013: The troposphere-to-stratosphere transition in kinetic energy spectra and nonlinear spectral fluxes as seen in ECMWF analyses. J. Atmos. Sci., 70, 669-687, https://doi.org/10.1175/JASD-12-0129.1.

Cullen, M. J. P., 2017: The impact of high vertical resolution in the Met Office Unified Model. Quart. J. Roy. Meteor. Soc., 143, 278-287, https://doi.org/10.1002/qj.2920.

Gill, A. E., 1982: Ocean-Atmosphere Dynamics. Academic Press, $662 \mathrm{pp}$.

Guest, F. M., M. J. Reeder, C. J. Marks, and D. J. Karoly, 2000: Inertia-gravity waves observed in the lower stratosphere over Macquariae Island. J. Atmos. Sci., 57, 737752, https://doi.org/10.1175/1520-0469(2000)057<0737: IGWOIT>2.0.CO;2.

Hamilton, K., Y. Takahasi, and W. Ohfuchi, 2008: Mesoscale spectrum of atmospheric motions investigated in a very fine resolution global general circulation model. J. Geophys. Res., 113, D18110, https://doi.org/10.1029/2008JD009785.

Hertzog, A., F. Vial, C. R. Mechoso, C. Basdevant, and P. Cocquerez, 2002: Quasi-Lagrangian measurements in the lower stratosphere reveal an energy peak associated with near-inertial waves. Geophys. Res. Lett., 29, 1229, https:// doi.org/10.1029/2001GL014083.

Hong, S.-Y., Y. Noh, and J. Dudhia, 2006: A new vertical diffusion package with an explicit treatment of entrainment processes. Mon. Wea. Rev., 134, 2318-2341, https://doi.org/ 10.1175/MWR3199.1.

Iga, S.-I., H. Tomita, M. Satoh, and K. Goto, 2007: Mountainwave-like spurious waves associated with simulated cold fronts due to inconsistencies between horizontal and vertical resolutions. Mon. Wea. Rev., 135, 2629-2641, https:// doi.org/10.1175/MWR3423.1.

Klemp, J. B., 2017: Damping characteristics of horizontal Laplacian diffusion filters. Mon. Wea. Rev., 145, 4365-4379, https://doi.org/10.1175/MWR-D-17-0015.1.

— J. Dudhia, and A. D. Hassiotis, 2008: An upper gravity-wave absorbing layer for NWP applications. Mon. Wea. Rev., 136, 3987-4004, https://doi.org/10.1175/2008MWR2596.1.

Lean, H. W., and P. A. Clark, 2003: The effects of changing resolution on mesocale modelling of line convection and slantwise circulations in FASTEX IOP16. Quart. J. Roy. Meteor. Soc., 129, 2255-2278, https://doi.org/10.1256/qj.02.57. 
Lindborg, E., 1999: Can the atmospheric kinetic energy spectrum be explained by two-dimensional turbulence? J. Fluid Mech., 388, 259-288, https://doi.org/10.1017/S0022112099004851.

2005: The effect of rotation on the mesoscale energy cascade in the free atmosphere. Geophys. Res. Lett., 32, L01809, https://doi.org/10.1029/2004GL021319.

Lindzen, R. S., and M. Fox-Rabinovitz, 1989: Consistent vertical and horizontal resolution. Mon. Wea. Rev., 117, 2575-2583, https:// doi.org/10.1175/1520-0493(1989)117<2575:CVAHR>2.0.CO;2.

Malardel, S., and N. P. Wedi, 2016: How does subgrid-scale parametrization influence nonlinear spectral energy fluxes in global NWP models? J. Geophys. Res. Atmos., 121, 5395-5410, https://doi.org/10.1002/2015JD023970.

Nastrom, G., and K. Gage, 1985: A climatology of atmospheric wavenumber spectra of wind and temperature observed by commercial aircraft. J. Atmos. Sci., 42, 950-960, https://doi.org/ 10.1175/1520-0469(1985)042<0950:ACOAWS>2.0.CO;2.

Pecnick, N. J., and D. Keyser, 1989: The effect of spatial resolution on the simulation of upper-tropospheric frontogenesis using a sigma-coordinate primitive equation model. Meteor. Atmos. Phys., 40, 137-149, https://doi.org/10.1007/BF01032454.

Persson, P., and T. Warner, 1991: Model generation of spurious gravity waves due to inconsistency of the vertical and horizontal resolution. Mon. Wea. Rev., 119, 917-935, https://doi.org/ 10.1175/1520-0493(1991)119<0917:MGOSGW>2.0.CO;2.

Plougonven, R., and C. Snyder, 2007: Inertia-gravity waves spontaneously generated by jets and fronts. Part I: Different baroclinic life cycles. J. Atmos. Sci., 64, 2502-2520, https://doi.org/ 10.1175/JAS3953.1.

Sato, K., D. O'Sullivan, and T. Dunkerton, 1997: Low-frequency inertia-gravity waves in the stratosphere revealed by threeweek continuous observation with the MU radar. Geophys. Res. Lett., 24, 1739-1742, https://doi.org/10.1029/97GL01759.

_- T. Kumakura, and M. Takahashi, 1999: Gravity waves appearing in a high-resolution GCM simulation. J. Atmos. Sci., 56, 1005-1018, https://doi.org/10.1175/1520-0469(1999)056<1005: GWAIAH $>2.0 . \mathrm{CO} ; 2$.

Skamarock, W. C., 2004: Evaluating mesoscale NWP models using kinetic energy spectra. Mon. Wea. Rev., 132, 3019-3032, https://doi.org/10.1175/MWR2830.1.

_ , and J. B. Klemp, 2008: A time-split nonhydrostatic atmospheric model for weather research and forecasting applications.
J. Comput. Phys., 227, 3465-3485, https://doi.org/10.1016/ j.jcp.2007.01.037.

, M. G. Duda, L. D. Fowler, S.-H. Park, and T. Ringler, 2012: A multiscale nonhydrostatic atmospheric model using centroidal Voronoi tesselations and C-grid staggering. Mon. Wea. Rev., 140, 3090-3105, https://doi.org/10.1175/MWR-D11-00215.1.

— S.-H. Park, J. B. Klemp, and C. Snyder, 2014: Atmospheric kinetic energy spectra from global high-resolution nonhydrostatic simulations. J. Atmos. Sci., 71, 4369-4381, https:// doi.org/10.1175/JAS-D-14-0114.1.

Snyder, C., W. C. Skamarock, and R. Rotunno, 1993: Frontal dynamics near and following frontal collapse. J. Atmos. Sci., 50, 3194-3212, https://doi.org/10.1175/1520-0469(1993)050<3194: FDNAFF $>2.0 . \mathrm{CO} ; 2$.

Vaughan, G., and R. M. Worthington, 2007: Inertia-gravity waves observed by the UK MST radar. Quart. J. Roy. Meteor. Soc., 133, 179-188, https://doi.org/10.1002/qj.142.

Waite, M. L., 2016: Dependence of model energy spectra on vertical resolution. Mon. Wea. Rev., 144, 1407-1421, https:// doi.org/10.1175/MWR-D-15-0316.1.

— vortical motion. J. Fluid Mech., 517, 281-308, https://doi.org/ 10.1017/S0022112004000977.

—_ and — 2006: The transition from geostrophic to stratified turbulence. J. Fluid Mech., 568, 89-108, https://doi.org/ 10.1017/S0022112006002060.

— , and C. Snyder, 2009: The mesoscale kinetic energy spectrum of a baroclinic life cycle. J. Atmos. Sci., 66, 883-901, https:// doi.org/10.1175/2008JAS2829.1.

$\longrightarrow$, and - 2013: Mesoscale energy spectra of moist baroclinic waves. J. Atmos. Sci., 70, 1242-1256, https://doi.org/10.1175/ JAS-D-11-0347.1.

Wang, L., and M. J. Alexander, 2010: Global estimates of gravity wave parameters from GPS radio occultation temperature data. J. Geophys. Res., 115, D21122, https://doi.org/10.1029/ 2010JD013860.

Watanabe, S., K. Sato, Y. Kawatani, and M. Takahashi, 2015: Vertical resolution dependence of gravity wave momentum flux simulated by an atmospheric general circulation model. Geosci. Model Dev., 8, 1637-1644, https://doi.org/10.5194/ gmd-8-1637-2015. 